\title{
Second harmonic generation from strongly coupled localized and propagating phonon-polariton modes
}

\author{
Ilya Razdolski ${ }^{1,}{ }^{*}$ Nikolai Christian Passler, ${ }^{1}$ Christopher R. Gubbin,${ }^{2}$ Christopher \\ J. Winta, ${ }^{1}$ Robert Cernansky, ${ }^{2}$ Francesco Martini, ${ }^{2}$ Alberto Politi, ${ }^{2}$ Stefan \\ A. Maier, ${ }^{3,4}$ Martin Wolf, ${ }^{1}$ Alexander Paarmann, ${ }^{1}$ and Simone De Liberato ${ }^{2}$ \\ ${ }^{1}$ Fritz Haber Institute of the Max Planck Society, 14195 Berlin, Germany \\ ${ }^{2}$ School of Physics and Astronomy, University of Southampton, Southampton, SO17 1BJ, United Kingdom \\ ${ }^{3}$ Chair in Hybrid Nanosystems, Nanoinstitute Munich, Faculty of Physics, \\ Ludwig-Maximilians-Universität München, München, Germany \\ ${ }^{4}$ Department of Physics, Blackett Laboratory, Imperial College London, London SW7 2AZ, United Kingdom
}

(Dated: August 21, 2018)

\begin{abstract}
We experimentally investigate second harmonic generation from strongly coupled localized and propagative phonon polariton modes in arrays of silicon carbide nanopillars. Our results clearly demonstrate the hybrid nature of the system's eigenmodes and distinct manifestation of strong coupling in the linear and nonlinear response. While in linear reflectivity the intensity of the two strongly-coupled branches is essentially symmetric and well explained by their respective localized or propagative components, the second harmonic signal presents a strong asymmetry. Analyzing it in detail, we reveal the importance of interference effects between the nonlinear polarization terms originating in the bulk and in the phonon polariton modes, respectively.
\end{abstract}

Controlled confinement of light in sub-diffraction volumes has always been a key goal in photonics. In this regard, plasmonic systems provide rich opportunities for manipulation of light on the nanoscale ${ }^{1-3}$. Recently, an alternative approach utilizing polar dielectrics operating in the mid-infrared (mid-IR) spectral range has attracted considerable attention $^{4-14}$. The photonic modes of these systems, termed surface phonon-polaritons, exhibit low optical losses due to their relatively large phonon lifetimes, allowing for higher degrees of energy concentration and smaller Purcell factors than their plasmonic counterparts ${ }^{15}$. Conventional methods of probing the field enhancement include multi-photon effects such as surface enhanced Raman scattering, optical rectification, and second harmonic generation (SHG).

Bearing strong conceptual similarities to nonlinear plasmonics in metals ${ }^{16}$, efficient SHG mediated by localized and propagating phonon-polariton modes in polar dielectrics has been reported ${ }^{17-20}$. In contrast to noble metals supporting surface plasmon-polaritons, the lack of inversion symmetry enables bulk SHG in these systems, opening intriguing questions about the nonlinearoptical response of phonon-polaritons. In 2016 Gubbin and coworkers observed strong coupling between phononpolariton modes localised in SiC nanopillars and propagative modes sustained on the substrate surface, giving rise to the two hybrid localised-propagative polariton branches ${ }^{8}$. While the mechanisms of SHG enhancement in plasmonic and phononic structures are reasonably well understood, the nonlinearity of coupled systems where multiple interacting resonances hybridize remains widely unexplored. Previous works on coupled localized and propagating plasmon modes ${ }^{21-27}$ focused on the linear optical response and its tunability prospects, meaning that little is known about the nonlinear-optical properties of the coupled polaritonic modes.
In this Article, we study the nonlinear-optical response of coupled surface phonon-polaritons in arrays of $\mathrm{SiC}$ nanopillars. Employing a free-electron laser (FEL) as a powerful, tunable source of mid-IR radiation we perform SHG spectroscopy on a series of samples with varied array pitch, thus tuning the surface phonon-polariton resonance. Our results demonstrate pronounced differences between the spectral reflectivity and SHG response at the avoided crossing in the polariton dispersion. We further outline relevant mechanisms for the observed SHG spectral behaviour and discuss the key role of the substrate for the disparate optical response of the coupled modes in the linear and nonlinear domains.

We perform spectroscopic measurements in the IR range, recording both linear reflectivity and SHG from square arrays of $\mathrm{SiC}$ nanopillars $(0.8 \mu \mathrm{m}$ high, $1 \mu \mathrm{m}$ in diameter) etched on a $3 \mathrm{C}$-SiC substrate. The fabrication procedure has previously been described in the literature $^{8}$. The FEL radiation $(10 \mathrm{~Hz}$ macropulse repetition rate, 3 ps micropulse duration, $5 \mathrm{~cm}^{-1}$ bandwidth, see Ref. ${ }^{28}$ ) was tuned through a frequency range of $750-1050 \mathrm{~cm}^{-1}$ (wavelength $9-13 \mu \mathrm{m}$ ). The fundamental $p$-polarised beam of about $10^{2} \mathrm{~W}$ average power during the macropulse ( $\sim 10 \mu \mathrm{J}$ micropulse energy) was focused onto the sample into a spot of about $200 \mu \mathrm{m}$ in diameter by means of $\mathrm{Au}$ spherical mirrors with a focal distance of about $f=15 \mathrm{~cm}$. The angle of incidence was $\theta=62^{\circ}$ from the surface normal. The outgoing SHG radiation was collected by another 2 -inch spherical $\mathrm{Au}$ mirror at the $2 f$ distance and directed onto a liquid nitrogen-cooled mercury cadmium telluride detector (Infrared Associates). The long-pass $7 \mu \mathrm{m}$ filter (LOT) in the incident beam ensured the suppression of intrinsic FEL-produced harmonics, whereas the fundamental frequency was filtered out from the SHG output by means of a $\mathrm{MgF}_{2}$ plate. The reflected fundamental radiation was 
registered by a home-built pyroelectric photodetector.

Localized phonon polaritons are supported in the individual nanopillars ${ }^{4,19,29}$, while propagative surface phonon polaritons are supported by the $\mathrm{SiC}$ substrate $^{20,30,31}$. The periodicity of the nanopillars enables phase-matched coupling of far field, p-polarised radiation into the propagative modes ${ }^{32,33}$ which can hybridize with the localized modes of the pillars ${ }^{8,34}$. These modes are observed as narrow dips on the high Reststrahlen reflectivity background of the substrate, as well as pronounced peaks in the SHG spectra due to strong light localization and enhancement of the electromagnetic field. Typical spectra shown in Fig. 1a were obtained on a sample with the array pitch $d=5.5 \mu \mathrm{m}$. As a few localized polariton modes observed inside the Reststrahlen band have been analysed in a number of previous publications $s^{4,8,19,29}$, here we directly employ these results to identify the observed spectral features. The monopole mode appears at about $865 \mathrm{~cm}^{-1}$ while the frequency of the propagative phonon-polariton (here $\approx 890 \mathrm{~cm}^{-1}$ ) shifts with the array pitch. The positions of the features associated with the excitation of surface phonon resonances are indicated with the dashed vertical lines. Note that at this particular array pitch, the coupling between the localized and propagative polariton modes is weak, allowing the observation of the two fundamental modes in an almost unperturbed regime. In what follows, we shall focus on those features and do not discuss the dipole localized SPhP modes, as well as the SHG peaks at the frequencies of the transverse $\left(\approx 790 \mathrm{~cm}^{-1}\right)$ and longitudinal $\left(\approx 980 \mathrm{~cm}^{-1}\right)$ optical phonons in $\mathrm{SiC}$, which are the fingerprints of the $\mathrm{SiC}$ crystalline symmetry. These features have been analyzed previously ${ }^{35,36}$ and are insensitive to the pitch $d$ of the nanopillar array.

Strong coupling of the polaritonic modes can be observed if the array pitch enables the excitation of propagating surface phonon polaritons at a frequency near to that of a localized mode ${ }^{8}$. In particular, this results in significant hybridization of the propagating phononpolariton with a so-called monopolar mode ${ }^{4,29,34}$ accompanied with the avoided crossing behaviour. To study the strong coupling regime in detail and map out the dispersion of the coupled modes in the vicinity of the avoided crossing, we performed spectroscopic measurements on a series of arrays with varied pitch $d$ in the range of $5.3-6.6 \mu \mathrm{m}$. The reflectivity data (Fig. 1b) are in a good agreement with the previously published results $^{8,34}$, demonstrating the avoided crossing of the dispersion curves of the two phonon-polariton modes. As in this work we are mostly interested in the nonlinearoptical response of the coupled polaritonic modes, we compare the SHG spatio-spectral map with that in the linear domain. The direct comparison (Fig. 2) reveals similar behaviour of the dispersion of the coupled modes. Further, the coupling of the propagating surface mode to the monopolar localized phonon-polariton results in noticeable spectral shifts of the linear extinction and SHG output peaks, demonstrating that the nonlinear emission
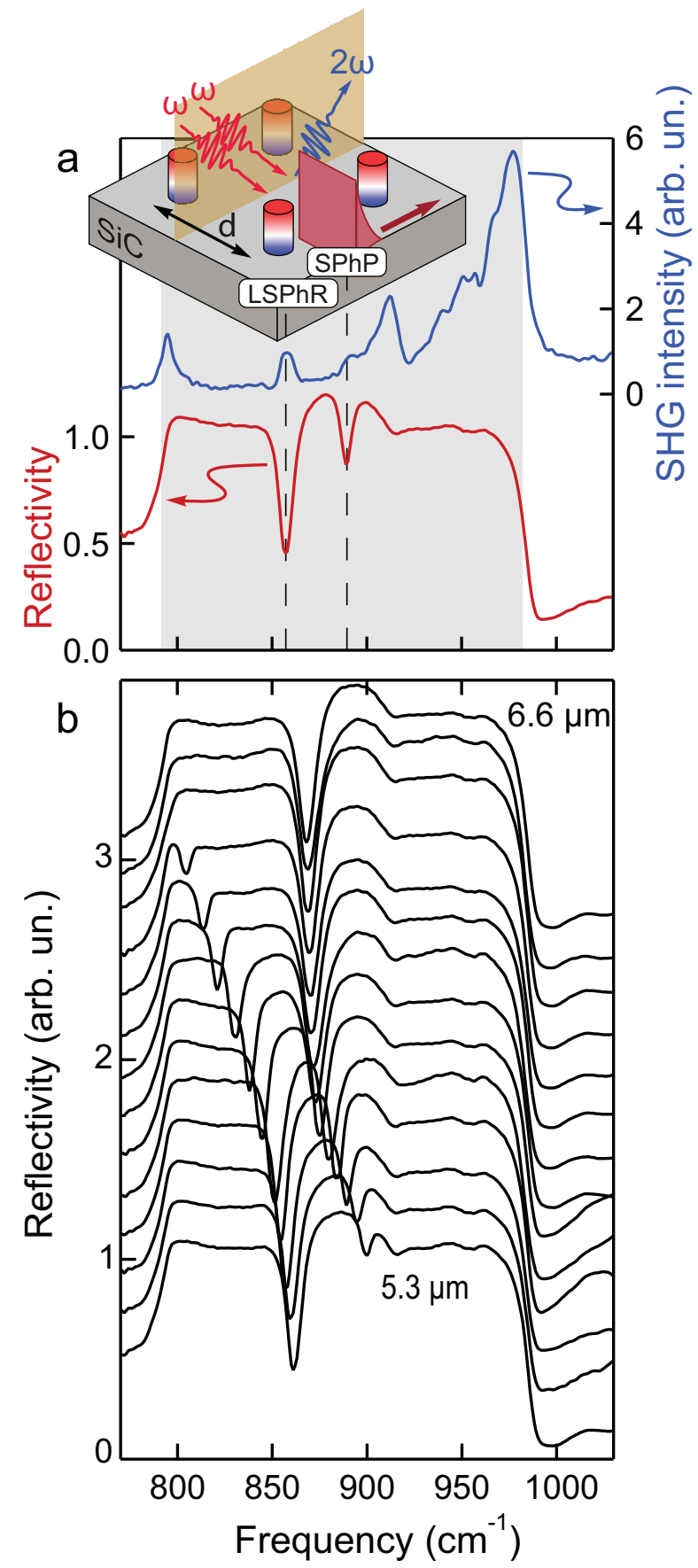

Figure 1. Strong coupling of a localized surface phonon resonance (LSPhR) and a propagating surface phonon-polariton (SPhP) mode. (a) Typical linear reflectivity (red) and SHG intensity (blue) spectra measured on a nanopillar array with pitch $d=5.5 \mu \mathrm{m}$. The vertical dashed lines indicate the positions of the surface phonon resonances. The gray box shows the $\mathrm{SiC}$ Reststrahlen band. Inset: sketch of the experimental geometry and the excited propagating and monopole localized polariton modes. (b) Linear reflectivity plots measured on a series of arrays showing the shift of the resonances with varied pitch due to the localised-propagative mode coupling. 

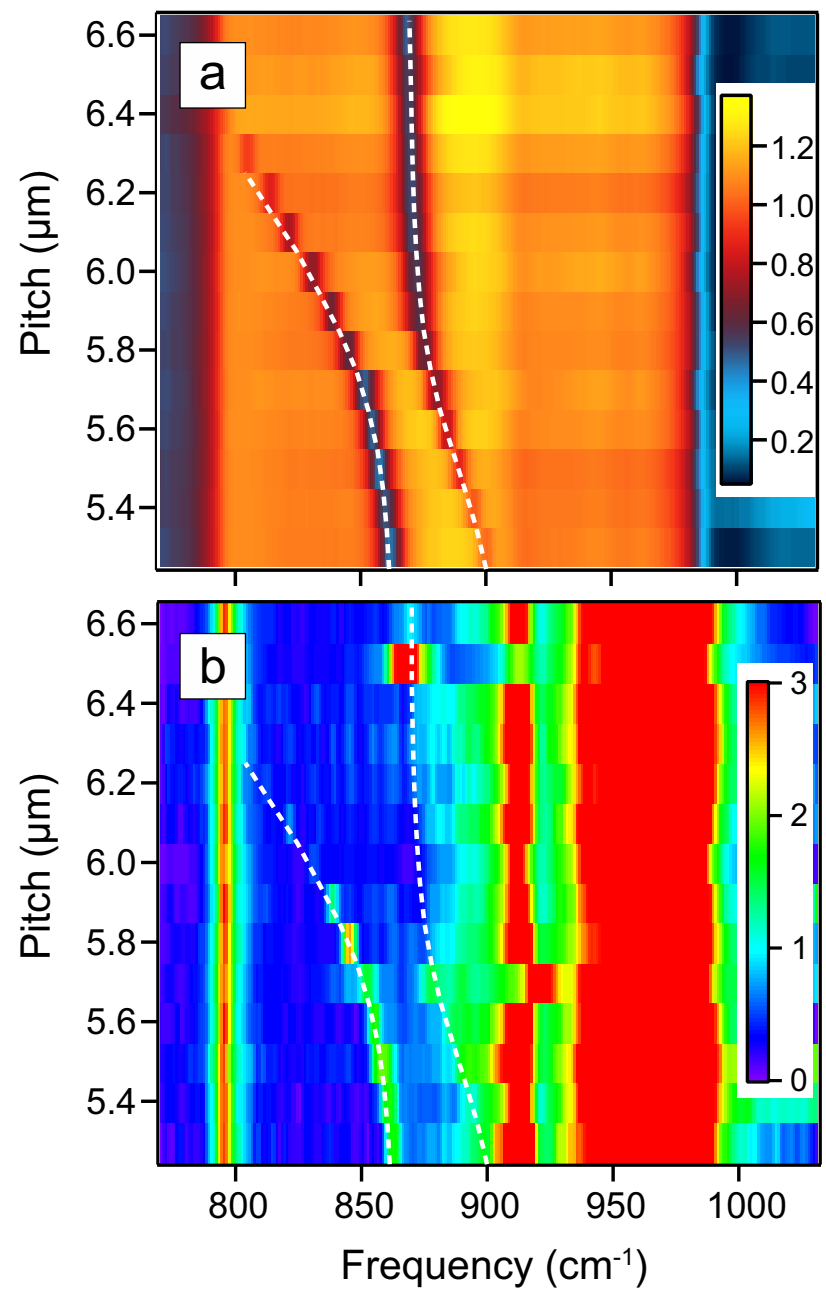

Figure 2. False color experimental spatio-spectral maps of linear reflectivity (a) and SHG intensity (b) measured on a set of arrays of nanopillars with varied pitches $d$. The white dashed lines illustrate the calculated dispersion of the strongly coupled polariton modes.

originates in the hybrid localized-propagative modes.

Notably, in Fig. 2b it is clearly seen that in the SHG output the low-frequency polariton branch is more strongly pronounced than the high-frequency one, while in the reflectivity map the two branches are almost symmetric. The latter means here that along both branches, the reflectivity depth is the largest at the monopolar localized mode and gradually decreases upon moving away from it. The difference in the slopes of the two branches seen here and in Fig. 3a originates in the $\mathrm{SiC}$ dispersion and will not be discussed further. To better understand the difference in the linear reflectivity and SHG output spectra we analysed the magnitudes of the SHG peaks and the reflectivity dips along the dispersion curves modified by the strong coupling. In order to do this, for each pitch $d$ we extracted the reflectivity and SHG data at the frequencies given by the dispersion of the coupled modes $\omega^{ \pm}$:

$$
\omega^{ \pm}=\frac{\omega_{1}+\omega_{2} \pm \sqrt{\left(\omega_{1}-\omega_{2}\right)^{2}+4 g^{2}}}{2},
$$

where $\omega_{1,2}$ are the frequencies of the localized and propagative modes, and $g$ is the coupling strength (Rabi frequency). The results of this procedure are shown in Fig. 3, where the open and full symbols refer to the upper $\left(\omega^{+}\right)$and lower $\left(\omega^{-}\right)$polariton branches, respectively.

In Fig. 3a we plot the depth of the corresponding dip in the reflectivity, that is, the difference between the high reflectance within the Reststrahlen band observed on a flat $\mathrm{SiC}$ surface and the respective data point obtained on the array of nanopillars. This difference is taken at the frequencies $\omega^{ \pm}$given by the calculated dispersion of the coupled modes (with $g \approx 16 \mathrm{~cm}^{-1}$ ), shown in Fig. 2 with white dashed lines. Each pair of points (consisting of an open and a closed symbol) in Fig. 3 corresponds to a particular array pitch $d$. The pronounced increase of the depth upon approaching the frequency of the monopolar mode $\omega_{1} \approx 865 \mathrm{~cm}^{-1}$ is related to the hybrid character of the two coupled modes. While the monopolar localized mode couples strongly to the far-field radiation, suboptimal grating conditions mean that the excitation of the surface phonon-polariton with free-space photons is inefficient. This inequality results in deeper reflectivity dips when a hybrid mode is predominantly monopolar in character, as it can be seen in Fig. 1. The reflectivity dip depth of the monopolar mode (around $865 \mathrm{~cm}^{-1}$ ) is significantly larger than the depth at the propagative mode far away from it. When the detuning of the bare, uncoupled polaritons approaches zero, their hybridization results in the coupled modes comprised of approximately equal proportions of monopolar localized and propagative modes, leading to the equilibration of the depths of the resonances. The slight asymmetry visible in the reflectivity data (Fig. 3a) can be attributed to the upper polariton (at frequency $\omega^{+}$) engaging in a quasi-resonance with the localized transverse dipolar mode at around $912 \mathrm{~cm}^{-1}$. The resulting avoided coupling slightly red-shifts the upper polariton branch, leading to the small difference in the slopes for the two polaritonic modes.

However, the hybridization between the two disparate polariton modes upon their strong coupling alone cannot account for the striking asymmetry in the SHG intensity from the coupled modes. It is seen in Fig. 3b that the SHG output along the lower polariton branch increases upon approaching the monopolar mode from the low frequency end, whereas the upper branch shows the opposite trend. The larger SHG output of the propagative mode on the high-frequency end of the spectrum $\left(\approx 900 \mathrm{~cm}^{-1}\right)$ compared to that on the opposite side $\left(\approx 820 \mathrm{~cm}^{-1}\right)$ originates in the interplay of the dispersion of the SiC nonlinear susceptibility $\chi^{(2)}$ and the effective Fresnel factors for the electric field including its polariton-driven enhancement ${ }^{10,36}$. The former factor peaks at the transverse optical phonon resonance at 


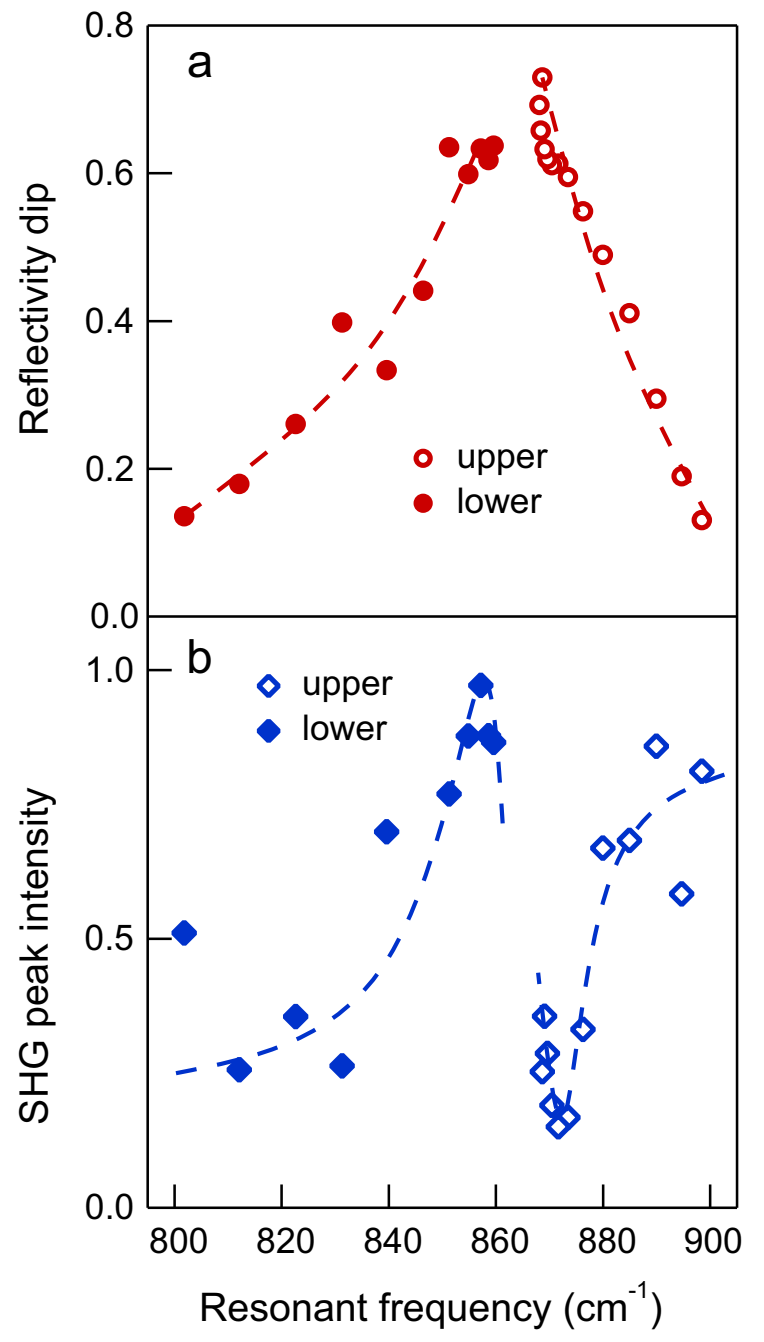

Figure 3. Analysis of the coupled polariton modes. Magnitudes of the reflectivity dip (a) and peak SHG intensity (b) along the dispersion branches of the strongly coupled polariton modes. The dashed lines are guides to the eye.

about $780 \mathrm{~cm}^{-1}$, whereas the latter is responsible for the SHG peak at the longitudinal optical phonon frequency $\left(\approx 980 \mathrm{~cm}^{-1}\right)$. The characteristic SHG spectrum shown in Fig. 1a, consistent with previous results of $\mathrm{SiC}$ SHG spectroscopy ${ }^{35,36}$, indicates the higher importance of the electric field enhancement factor, giving rise to the steadily increasing SHG background at larger frequencies in the spectral range of $830-950 \mathrm{~cm}^{-1}$.

Summarizing these observations, we note that the SHG spectrum of the coupled polaritons demonstrates strikingly disparate behaviour to the relatively simple and intuitive picture obtained in the linear reflectivity measurements. To understand this difference, we employ a simple model of two coupled oscillators giving rise to the SHG signal on top of the background contribution. For clarity, we neglect the weak interaction of the propagative polariton with the aforementioned transverse dipolar localized mode. Within this model, the resonances can be described by the two Lorentzians:

$$
L_{k}(\omega)=\frac{A_{k}}{\omega-\omega_{k}+i \gamma_{k}} e^{i \varphi_{k}},
$$

where $k=1,2, \omega_{k}, \gamma_{k}$ and $\varphi_{k}$ are the resonant frequencies, damping rates, and phases of the two modes. $A_{k}$ are the effective oscillator strengths of the two modes related to the their excitation efficiency. In our model, $\omega_{1}$ is set constant to $865 \mathrm{~cm}^{-1}$ (to match the monopole mode frequency) while $\omega_{2}$ can be tuned by an external parameter, i.e., the array pitch $d$, representing the dispersion of the propagative mode and its grating-mediated excitation. To simulate the propagative-monopole mode coupling, we continuously change the control parameter $d$ so that $\omega_{2}$ sweeps across $\omega_{1}$, and the resonant frequencies of the coupled modes are given by Eq. 1 (see Fig. 4a). There, the extinction of the electric field $E(\omega)$ is given by the imaginary part of the linear-optical response at the frequencies $\omega^{ \pm}$:

$$
r\left(\omega^{ \pm}\right) \propto \operatorname{Im}\left[L_{1}\left(\omega^{ \pm}\right)+L_{2}\left(\omega^{ \pm}\right)\right]
$$

where the permutation of $\omega^{ \pm}$does not change the result. In turn, the depth in the experimental reflectivity data is proportional to $\left|r\left(\omega^{ \pm}\right)\right|^{2}$ (Fig. 4b). Here we neglect the absorption in the $\mathrm{SiC}$ substrate due to its high reflectivity within the Reststrahlen band.

On the contrary, the SHG response needs to include the background contribution of the substrate. We can write down the nonlinear polarization at the double frequency $P^{2 \omega}$ created by the excitation at the fundamental frequency $\omega$ in the following way:

$$
P^{2 \omega}\left(\omega^{ \pm}\right) \propto \chi_{1}^{(2)} L_{1}^{2}\left(\omega^{ \pm}\right)+\chi_{2}^{(2)} L_{2}^{2}\left(\omega^{ \pm}\right)+A_{\mathrm{bg}} e^{i \varphi_{\mathrm{bg}}} .
$$

Here $\chi_{k}^{(2)}$ are the effective nonlinear-optical susceptibilities of the two modes, and we have introduced a background nonlinearity with its amplitude $A_{\mathrm{bg}}$ and phase $\varphi_{\mathrm{bg}}$. The resonant increase of the SHG efficiency is attributed to the the polariton-induced enhancement of the electric field $E \propto L_{k}$. For instance, the SHG enhancement at the localized monopolar mode originates in the subdiffractional localization of the electric field and the prominent increase of its out-of-plane component $E_{z}{ }^{10,19}$. The surface-mode-induced SHG is additionally quenched by the low efficiency of the grating consisting of the nanopillars, as discussed above. Within our model, the experimentally observed SHG intensities at the frequencies of the coupled modes are given by $\left|P^{2 \omega}\left(\omega^{ \pm}\right)\right|^{2}$.

The results of our modeling are summarized in Fig. 4, nicely resembling our experimental observations. The slight asymmetry between the two branches in the reflectivity (Fig. 4b) is introduced by the phase shift between the localized and propagative resonances, $\Delta \varphi=\varphi_{2}-\varphi_{1}$. We found that the degree of asymmetry commensurate with the experimental observations can be obtained for 


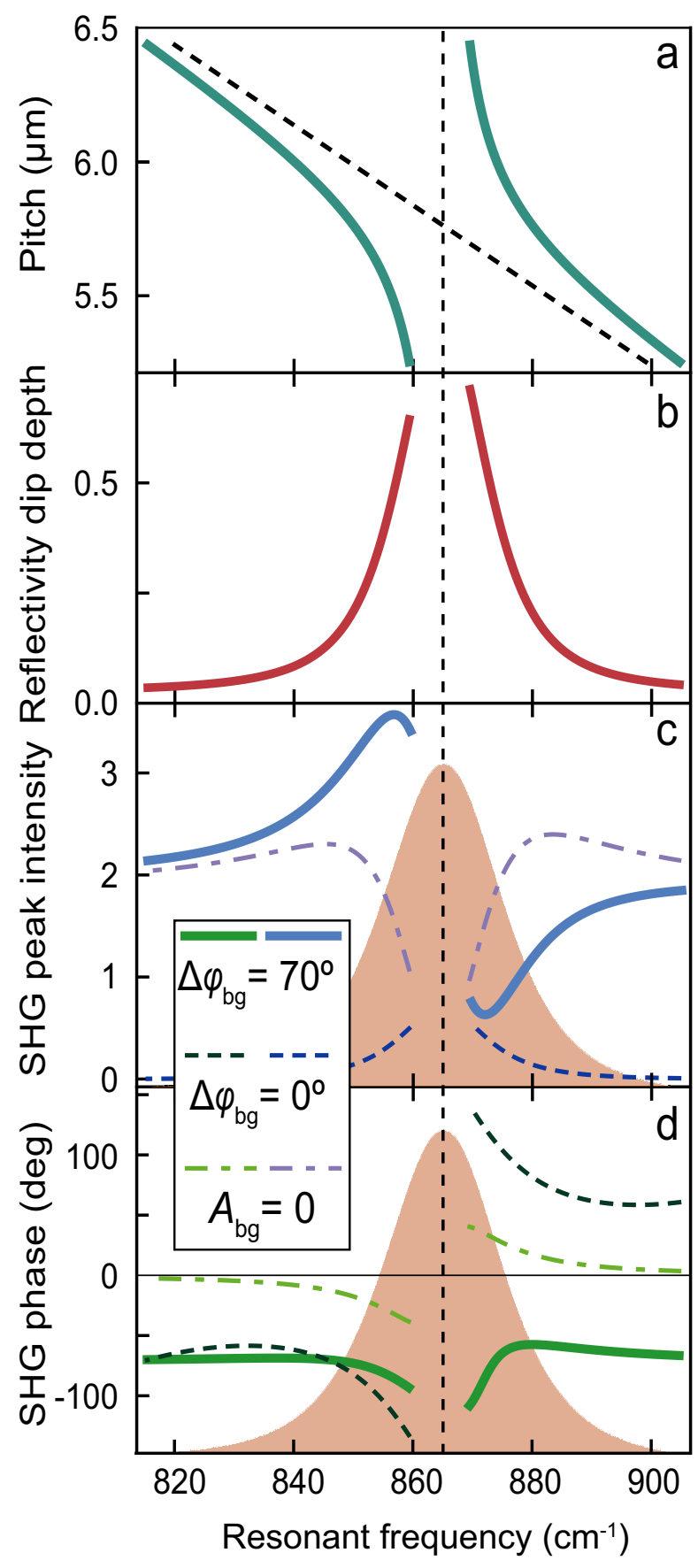

Figure 4. Two coupled oscillators model. (a) Dispersion of the uncoupled (dashed black lines) and the strongly coupled modes (solid green lines). (b,c) Calculated reflectivity dip (b, solid red lines) and SHG peak intensity (c, solid blue lines) along the branches of the coupled modes. The shaded area in (c) illustrates the lineshape of a localized oscillator. (d) The calculated phase of the nonlinear polarization $P^{2 \omega}$ in the vicinity of the two resonances. The solid lines in (c-d) were calculated with a phase of the complex background contribution $A_{\mathrm{bg}} \varphi_{\mathrm{bg}}=70^{\circ}$ with respect to that of the resonant Lorentzians (Eq. 2), resembling the experimental observations. Such agreement cannot be met when $A_{\mathrm{bg}}$ is set to have the same phase as $L_{k}$, or $A_{\mathrm{bg}}=0$, as is illustrated by the dashed and dot-dashed lines, respectively.
$|\Delta \varphi|<5^{\circ}$. Taking into account the simplicity of our model and possible quasi-resonance with the transverse dipolar polariton, we can conclude on the zero phase shift between the two considered polariton modes. At the same time, the phase shift between the resonant and the background SHG contributions appears crucial for the asymmetry in the SHG dependence. The antisymmetric shape seen in Fig. 4c can only be obtained when the phase shift of the complex background contribution $A_{\mathrm{bg}}$ with respect to the resonant Lorentzians $\left|\Delta \varphi_{\mathrm{bg}}\right|=\left|\varphi_{\mathrm{bg}}-\varphi_{1,2}\right|$ is set to about $90^{\circ}$. In particular, the results of our calculations shown with the solid lines in Fig. 4c were obtained for $\Delta \varphi_{\mathrm{bg}} \approx 70^{\circ}$.

To demonstrate the importance of the phase-shifted background contribution to the total SHG output in the vicinity of the strong coupling, in Fig. 4c we further plot the shapes of the resonant SHG yields if the phase shift $\Delta \varphi_{\mathrm{bg}}$ vanishes (dashed lines) and if the background contribution is not taken into account at all (dash-dotted lines). It is seen that the experimentally observed degree of asymmetry in the resonant SHG response can only be obtained in calculations if the background contribution $A_{\mathrm{bg}}$ with its own distinct phase is considered. If either $\varphi_{\mathrm{bg}}$ or the entire $A_{\mathrm{bg}}$ is set to zero, it is further seen that when $|\Delta \varphi| \approx 0$ (as enforced by the linear reflectivity data), the asymmetry of the two branches of the coupled resonances is eventually lost, thus ruling out the reproduction of the experimentally achieved SHG behaviour. Fig. 4d provides additional insight into the nonlinear optics of the strongly coupled resonances, showing the calculated phases of the nonlinear polarization $P^{2 \omega}$ in these three cases. The curves in Fig. 4 were calculated for a coupling strength of $g=15 \mathrm{~cm}^{-1}$, which is in a good agreement with the values found from the analysis of the experimental data ${ }^{8}$. There, linear reflectivity was recorded in similar nanopillar arrays, and then the dispersion of the coupled polariton modes was retrieved from the fits of the reflectivity spectra, indicating strong coupling of the localized and propagative polaritons in comparable experimental conditions.

As such, this disparate behaviour of the resonant linear and nonlinear properties is an interesting optical phenomenon originating in the utter complexity of the nonlinear-optical response ${ }^{37,38}$. We emphasize that the goal of our modelling was not to accurately reproduce the experimental data (which would require inclusion of too many fitting parameters in the model) but rather to demonstrate how the main features of the SHG intensity variations in the vicinity of the coupled resonances, which exhibit drastically different behaviour to that of the linear reflectivity data, can be relatively simply understood. With the help of our modelling we found that the interference of the resonantly enhanced SHG waves with the phase-shifted background contribution produced by the substrate is a key for the asymmetry between the two branches of the coupled polaritonic modes. Importantly, this phase shift is inherent to the polaritonic nature of the modes: on top of the well-known enhancement of the 
electric field amplitude, the surface polaritons (both localized and propagating) are characterized by the phases of various electric field components. In other words, the phase shift of the electric fields associated with the particular surface polariton mode is then imprinted into the phase of the corresponding nonlinear polarization term $P^{2 \omega}$. Because our model does not explicitly consider the polariton-driven electric field enhancement, this effect is instead taken into account by introducing the effective phases $\varphi_{k}$ and susceptibilities $\chi_{k}^{(2)}$. As such, not only the amplitude but also the phase of the resulting total $P^{2 \omega}$ can vary between the resonant and non-resonant cases, giving rise to the interesting interference conditions at the strong coupling resonance.

To summarize, we have analyzed the SHG response of strongly coupled surface phonon-polariton modes. In particular, we observe SHG from the normal modes of an array of $\mathrm{SiC}$ nanoresonators, consisting of hybridized localized and propagating surface phonon-polaritons in the SiC Reststrahlen band. The far-field excitation of the polaritons is enabled by the periodicity of $\mathrm{SiC}$ nanopillar arrays with a systematically varied pitch. In contrast to the linear reflectivity measurements, we found a clear antisymmetric behaviour of the resonant SHG output along the two dispersion branches of the coupled polaritons. Employing a simple coupled oscillator model, we demonstrate that the disparate symmetry of the linear and SHG response can be explained by the interference of the polariton-induced SHG with the background contribution. We further argue that the polaritonic enhancement of the electric fields and their phase shifts are responsible for the particular interference conditions leading to the experimentally observed asymmetry. Our results advance the understanding of the nonlinear nanophononics in the mid-infrared spectral range, while retaining a high degree of generality and thus remaining valid for e.g. surface plasmon-polaritons in metals.

\section{ACKNOWLEDGMENTS}

The authors thank W. Schöllkopf and S. Gewinner for their assistance with operating the free electron laser. S.D.L. is a Royal Society Research Fellow. S.D.L and C.R.G. acknowledge support from EPSRC Grant No. EP/M003183/1. S.A.M. acknowledges the Lee-Lucas Chair in Physics and the Leverhulme Trust.

\section{APPENDIX: MATHEMATICAL FORMALISM}

We begin with a model of two coupled oscillators with eigenfrequencies $\omega_{1,2}$ and a coupling strength $g$. There are multiple ways to obtain the frequencies of the coupled modes $\omega^{ \pm}$. Here we use a relatively straightforward Hopfield approach, where in the rotating wave approximation the Hamiltonian $\mathcal{H}$ takes the following form ${ }^{8,39,40}$ :

$$
\mathcal{H}=\hbar \omega_{1} \hat{a}^{\dagger} \hat{a}+\hbar \omega_{2} \hat{b}^{\dagger} \hat{b}+\hbar g\left(\hat{a}^{\dagger} \hat{b}+\hat{a} \hat{b}^{\dagger}\right)
$$

where the $\hat{a}^{\dagger}(\hat{a})$ and $\hat{b}^{\dagger}(\hat{b})$ are the bosonic creation (annihilation) operators for the two uncoupled oscillators. The coupling strength $g$ is introduced as an effective phenomenological parameter. The eigenfrequencies of the coupled modes can be obtained by diagonalizing the Hopfield-Bogoliubov matrix ${ }^{8}$ :

$$
H=\left(\begin{array}{cc}
\omega_{1} & g \\
g & \omega_{2}
\end{array}\right)
$$

The eigenvalues of this matrix yields the frequencies $\omega^{ \pm}$ from Eq. (1).

Next, we approximate the spectral lineshapes of the two resonances with Lorentzians (2) with their amplitudes $A_{k}$, frequencies $\omega_{k}$ and damping rates $\gamma_{k}$. Then, the optical losses due to absorption are intertwined with the mode excitation efficiency and given by the imaginary part of the Lorentzians, $\operatorname{Im~} \mathrm{L}(\omega)$. Assuming the weak nonlinear-optical conversion, the optical absorption at the fundamental frequency is linear and thus can be represented as a sum of absorption coefficients provided by the two modes independently. In the case of the coupled modes, optical losses (or extinction) at the eigenfrequencies $\omega^{ \pm}$is thus given by Eq. (3).

In SHG, the nonlinear (second-order) polarization is given by:

$$
P^{2 \omega}=\chi^{(2)}: E^{\omega} E^{\omega}
$$

where $\chi^{(2)}$ is the nonlinear susceptibility tensor. The excitation of a polariton mode results in a prominent enhancement of the fundamental electric field $E^{\omega}$, which can be taken into account in Eq. (7) by introducing the so-called local field factors $L^{\omega}$. The latter relate the electric fields in the nonlinear medium $E^{\omega}$ from Eq. (7) to the incident optical fields $E_{\text {in }}^{\omega}$ :

$$
E^{\omega}=L^{\omega} E_{\mathrm{in}}^{\omega}
$$

Peaking at the resonance frequencies, the lineshapes of these local factors $L^{\omega}$ can be modeled with the same Lorentzians $L(\omega)$ as the optical extinction. Additionally, the possible phase shift of the electric fields due to the polaritonic mode nature is accounted for by multiplying $L_{k}(\omega)$ with a phase term $e^{i \varphi_{k}}$. Note that this is the effective phase originating in the tensorial character of the nonlinear susceptibility $\chi^{(2)}$. To illustrate it, we consider a simple example of a propagative polariton at an isotropic interface. There, three independent non-zero $\chi^{(2)}$ components exist $\left(\chi_{\perp \perp \perp}^{(2)}, \chi_{\perp\|\| \|}^{(2)}\right.$, and $\left.\chi_{\|\perp\|}^{(2)}\right)$. The total nonlinear polarization is given by the interference of the three respective terms of $\chi_{i j k}^{(2)} E_{j} E_{k}$, and the phases 
of the electric fields $E_{j, k}$ are imprinted into the phase of the respective $P^{2 \omega}$ term. Because it is not practical to introduce the phases and amplitudes of each $\chi_{i j k}^{(2)}$ component and each field projection $E_{j, k}$, we instead consider effective phases and amplitudes only.

Employing these effective parameters, we can write:

$$
P^{2 \omega}=\chi^{(2)}: L(\omega) L(\omega) e^{i 2 \varphi},
$$

which in the case of the two coupled polaritons and the background contribution can be expanded into Eq. (4). Further, because we are not interested in the absolute values of the SHG intensity, we can write the proportionality:

$$
I^{2 \omega} \propto\left|P^{2 \omega}\right|^{2}
$$

Since the total phase of the nonlinear polarization is not registered in the experiment, the common phase of the three terms in Eq. (4) can be eliminated, and the number of phase parameters is effectively reduced to two, namely, the phase difference between the two resonances, $\Delta \varphi=\varphi_{2}-\varphi_{1}$, and the relative phase of the background contribution $\Delta \varphi_{\mathrm{bg}}=\varphi_{\mathrm{bg}}-\varphi_{1}$. Since the analysis of our experimental results requires $\Delta \varphi \approx 0$, in the definition of the background phase $\varphi_{1}$ can be substituted with $\varphi_{2}$ without the loss of generality.

To obtain the data shown in Fig. 4, we first calculate the resonant frequencies $\omega^{ \pm}$for each value of the array pitch $d$ (Fig. 4a). Then, using these values, we calculate the linear optical losses shown in Fig. 4b using Eq. (3) and plot them against the previously obtained resonant frequencies $\omega^{ \pm}$. Similarly, we plot the SHG intensity calculated with the help of Eqs. (4),(10) in Fig. 4c. The importance of the interference of the resonant SHG field with the background contribution can be seen in Fig. 4d, where the phase of the nonlinear polarization $P^{2 \omega}$ from Eq. (4) is shown. In the absence of the background contribution $\left(A_{\mathrm{bg}}=0\right.$, dash-dotted lines), the phase of $P^{2 \omega}$ is the opposite at the both sides of the localized polariton resonance. As such, interference with a phase-shifted background term with a frequency-independent amplitude results in the enhancement or suppression of the total SHG yield, in agreement with the experiment.
* razdolski@fhi-berlin.mpg.de

1 S. A. Maier, Plasmonics: Fundamentals and applications (Springer Science + Business Media, 2007).

2 T. W. Odom and G. C. Schatz, Chemical Reviews 111, 3667 (2011).

3 M. L. Brongersma, N. J. Halas, and P. Nordlander, Nature Nanotechnology 10, 25 (2015).

4 J. D. Caldwell, O. J. Glembocki, Y. Francescato, N. Sharac, V. Giannini, F. J. Bezares, J. P. Long, J. C. Owrutsky, I. Vurgaftman, J. G. Tischler, V. D. Wheeler, N. D. Bassim, L. M. Shirey, R. Kasica, and S. A. Maier, Nano Letters 13, 3690 (2013).

5 S. Dai, Z. Fei, Q. Ma, A. S. Rodin, M. Wagner, A. S. McLeod, M. K. Liu, W. Gannett, W. Regan, K. Watanabe, T. Taniguchi, M. Thiemens, G. Dominguez, A. H. C. Neto, A. Zettl, F. Keilmann, P. Jarillo-Herrero, M. M. Fogler, and D. N. Basov, Science 343, 1125 (2014).

6 J. D. Caldwell and K. S. Novoselov, Nature Materials 14, 364 (2015).

7 E. Yoxall, M. Schnell, A. Y. Nikitin, O. Txoperena, A. Woessner, M. B. Lundeberg, F. Casanova, L. E. Hueso, F. H. L. Koppens, and R. Hillenbrand, Nature Photonics 9, 674 (2015).

8 C. R. Gubbin, F. Martini, A. Politi, S. A. Maier, and S. De Liberato, Phys. Rev. Lett. 116, 246402 (2016).

9 P. Li, X. Yang, T. W. W. Ma, J. Hanss, M. Lewin, A.-K. U. Michel, M. Wuttig, and T. Taubner, Nature Materials 15, 870 (2016).

10 C. R. Gubbin and S. De Liberato, ACS Photonics 5, 284 (2018).

11 C. R. Gubbin and S. De Liberato, ACS Photonics 4, 1381 (2017).

12 N. C. Passler and A. Paarmann, J. Opt. Soc. Am. B 34, 2128 (2017).

13 A. D. Dunkelberger, C. T. Ellis, D. C. Ratchford, A. J.
Giles, M. Kim, C. S. Kim, B. T. Spann, I. Vurgaftman, J. G. Tischler, J. P. Long, O. J. Glembocki, J. C. Owrutsky, and J. D. Caldwell, Nature Photonics 12, 50 (2018).

14 R. Berte, C. Gubbin, V. Wheeler, A. Giles, V. Giannini, S. Maier, S. De Liberato, and J. Caldwell, ACS Photonics 5, 2807 (2018).

15 J. B. Khurgin, Nanophotonics 7, 305 (2017).

16 M. Kauranen and A. V. Zayats, Nature Photonics 6, 737 (2012).

17 E. Alieva, G. Zhizhin, V. Yakovlev, V. Sychugov, A. van der Meer, and M. van der Wiel, JETP Lett. 62, 794 (1995).

18 T. Dekorsy, V. A. Yakovlev, W. Seidel, M. Helm, and F. Keilmann, Phys. Rev. Lett. 90, 055508 (2003).

19 I. Razdolski, Y. Chen, A. J. Giles, S. Gewinner, W. Schöllkopf, M. Hong, M. Wolf, V. Giannini, J. D. Caldwell, S. A. Maier, and A. Paarmann, Nano Letters 16, 6954 (2016).

20 N. C. Passler, I. Razdolski, S. Gewinner, W. Schöllkopf, M. Wolf, and A. Paarmann, ACS Photonics 4, 1048 (2017).

21 W. R. Holland and D. G. Hall, Phys. Rev. Lett. 52, 1041 (1984).

22 Y. Borensztein, F. Abelès, and T. Lopez-Rios, Phys. Rev. Lett. 53, 854 (1984).

${ }^{23}$ H. R. Stuart and D. G. Hall, Phys. Rev. Lett. 80, 5663 (1998).

24 P. Nordlander and E. Prodan, Nano Letters 4, 2209 (2004).

25 A. Christ, T. Zentgraf, S. G. Tikhodeev, N. A. Gippius, J. Kuhl, and H. Giessen, Phys. Rev. B 74, 155435 (2006).

26 A. Ghoshal, I. Divliansky, and P. G. Kik, Applied Physics Letters 94, 171108 (2009).

27 M. Sarkar, M. Besbes, J. Moreau, J.-F. Bryche, A. Olivro, G. Barbillon, A.-L. Coutrot, B. Bartenlian, and M. Canva, ACS Photonics 2, 237 (2015). 
${ }^{28}$ W. Schöllkopf, S. Gewinner, H. Junkes, A. Paarmann, G. von Helden, H. P. Bluem, and A. M. M. Todd, Proc.SPIE 9512, 13 (2015).

29 Y. Chen, Y. Francescato, J. D. Caldwell, V. Giannini, T. W. W. Ma, O. J. Glembocki, F. J. Bezares, T. Taubner, R. Kasica, M. Hong, and S. A. Maier, ACS Photonics 1, 718 (2014).

30 A. Huber, N. Ocelic, D. Kazantsev, and R. Hillenbrand, Applied Physics Letters 87, 081103 (2005).

31 N. C. Passler, C. R. Gubbin, T. G. Folland, I. Razdolski, D. S. Katzer, D. F. Storm, M. Wolf, S. De Liberato, J. D. Caldwell, and A. Paarmann, Nano Letters 18, 4285 (2018).

32 J. Le Gall, M. Olivier, and J.-J. Greffet, Phys. Rev. B 55, 10105 (1997).

33 J.-J. Greffet, R. Carminati, K. Joulain, J.-P. Mulet,
S. Mainguy, and Y. Chen, Nature 416, 61 (2002).

34 C. R. Gubbin, S. A. Maier, and S. De Liberato, Phys. Rev. B 95, 035313 (2017).

35 A. Paarmann, I. Razdolski, A. Melnikov, S. Gewinner, W. Schöllkopf, and M. Wolf, Applied Physics Letters 107, 081101 (2015).

36 A. Paarmann, I. Razdolski, S. Gewinner, W. Schöllkopf, and M. Wolf, Phys. Rev. B 94, 134312 (2016).

37 T. Utikal, T. Zentgraf, T. Paul, C. Rockstuhl, F. Lederer, M. Lippitz, and H. Giessen, Phys. Rev. Lett. 106, 133901 (2011).

38 A. L. Chekhov, I. Razdolski, A. Kirilyuk, T. Rasing, A. I. Stognij, and T. V. Murzina, Phys. Rev. B 93, 161405 (2016).

39 J. Hopfield, Phys. Rev. 112, 1555 (1958).

40 V. Savona, Z. Hradil, A. Quattropani, and P. Schwendimann, Phys. Rev. B 49, 8774 (1994). 